\title{
Severe Respiratory Alkalosis in Acute Ischemic Stroke: A Rare Presentation
}

\author{
Vijayadershan Muppidi ${ }^{1}$, Sashank Kolli ${ }^{2}$, Vasuki Dandu ${ }^{3}$, Samata Pathireddy ${ }^{4}$, Sreenath Meegada ${ }^{5}$ \\ 1. Internal Medicine, Indiana University Health, Indianapolis, USA 2. Internal Medicine/Pulmonary and Critical Care, \\ Indiana University Ball Memorial Hospital, Muncie, USA 3. Neurology, Baptist Health Medical Center, Little Rock, USA \\ 4. Internal Medicine, Deaconess Health System/Indiana University School of Medicine, Evansville, USA 5. Internal \\ Medicine, The University of Texas Health Science Center/Christus Good Shepherd Medical Center, Longview, USA
}

Corresponding author: Vijayadershan Muppidi, vijaymuppidi@gmail.com

\begin{abstract}
Respiratory alkalosis is a rare but severe complication of acute ischemic stroke (AIS). In ischemic stroke, respiratory alkalosis results from hyperventilation due to the effect of stroke on the respiratory center. We report a case of a young male who presented with acute encephalopathy. Work-up revealed ischemic infarcts in the bilateral cerebellar and left posterior cerebral artery territory. Arterial blood gas (ABG) showed severe respiratory alkalosis with a $\mathrm{pH}$ of 7.72. Alkalosis resolved with mechanical ventilation. Such a high $\mathrm{pH}$ associated with AIS has not been reported in the medical literature so far. The index case highlights the severity of respiratory alkalosis that can be caused by an AIS. We conclude that early diagnosis and management of severe respiratory alkalosis is crucial for survival and recovery.
\end{abstract}

Categories: Internal Medicine, Neurology, Pulmonology

Keywords: acute cva, respiratory alkalosis, acute encephalopathy, cryptogenic stroke

\section{Introduction}

Stroke has a significant healthcare burden across the world. In 2009, one out of every 19 deaths in the United States was attributable to stroke [1]. According to the 2013 guidelines from the American Heart Association/American Stroke Association (AHA/ACC), acute ischemic stroke (AIS) is an episode of neurological dysfunction due to restriction of blood supply to a part of the central nervous system leading to the brain, spinal cord, retinal cell injury/death [2]. The cell injury usually happens in a defined vascular distribution and is recognized clinically, pathologically, or on imaging [2]. Clinical symptoms and the area of damage evident on imaging is dependent on the blood vessels involved. Common large arteries involved in ischemic stroke are anterior cerebral artery (ACA), middle cerebral artery (MCA), and posterior cerebral artery (PCA). The usual mechanisms of stroke are atherosclerotic and cardioembolic [3]. Stroke is classified as cryptogenic stroke if the cause is unknown or cannot be identified [4]. The lesions in brain due to stroke can cause hyperventilation leading to respiratory alkalosis [5]. Respiratory alkalosis is defined as a pH above 7.45 due to a pulmonary process [6]. We report a case of AIS that caused severe respiratory alkalosis with a

Received 04/13/2020

Review began 04/13/2020 Review ended 04/14/2020 Published 04/20/2020

\section{(c) Copyright 2020}

Muppidi et al. This is an open access article distributed under the terms of the Creative Commons Attribution License CC-BY 4.0., which permits unrestricted use, distribution, and reproduction in any medium, provided the original author and source are credited.
$\mathrm{pH}$ above 7.7, one of the highest $\mathrm{pH}$ associated with ischemic stroke reported in the medical literature so far.

\section{Case Presentation}

A 37-year-old male with a history of migraine, chronic back pain, seizure disorder, anxiety, and remote lower back spinal fusion surgery was brought to the hospital for headache, slurred speech, lethargy, visual hallucinations, and inability to get up from the floor. Symptoms started a day prior to presentation. His wife noticed mild drooping of the right side of his mouth. His wife endorsed nausea and vomiting. Home medications included hydrocodone-acetaminophen 5-325 mg every six hours as needed, gabapentin (dose unknown), and alprazolam (dose unknown). He never smoked, had occasional alcohol, and no history of illicit drug use. He works at a desk job for family-owned businesses.

On presentation, he was afebrile, had blood pressure (BP) 150/80 $\mathrm{mmHg}$, heart rate 53 beats/min, respiratory rate $18-26 / \mathrm{min}$, and $\mathrm{O} 2$ saturation $93 \%$ on room air. BMI was $32.4 \mathrm{~kg} / \mathrm{m} 2$. Glasgow Coma Scale was 14. Cardiac auscultation did not reveal a murmur. Neurological examination was significant for lethargy, intact motor and sensory systems and decreased reflexes in all four extremities. The patient was not cooperative to test cerebellar functions and gait. Initial laboratory work-up in the ER was unremarkable (Table 1). 


\section{Cureus}

\begin{tabular}{|c|c|c|}
\hline \multicolumn{2}{|l|}{ White blood cell count } & $10.6 \mathrm{k} /$ cumm \\
\hline \multicolumn{2}{|l|}{ Hemoglobin } & $14.9 \mathrm{gm} / \mathrm{dL}$ \\
\hline Hematocrit & \multicolumn{2}{|r|}{$42.4 \%$} \\
\hline Platelet count & \multicolumn{2}{|r|}{$333 \mathrm{k} /$ cumm } \\
\hline Sodium & & $140 \mathrm{mmol} / \mathrm{L}$ \\
\hline Potassium & & $4.2 \mathrm{mmol} / \mathrm{L}$ \\
\hline Chloride & & $105 \mathrm{mmol} / \mathrm{L}$ \\
\hline Bicarbonate & & $26 \mathrm{mmol} / \mathrm{L}$ \\
\hline Blood urea nitrogen & & $13 \mathrm{mg} / \mathrm{dL}$ \\
\hline Creatinine & & $0.89 \mathrm{mg} / \mathrm{dL}$ \\
\hline Glucose & & $136 \mathrm{mg} / \mathrm{dL}$ \\
\hline Anion Gap & & $9 \mathrm{mmol} / \mathrm{L}$ \\
\hline \multicolumn{2}{|l|}{ Alkaline phosphatase } & 84 units/L \\
\hline AST & & 34 units/L \\
\hline ALT & & 39 units/L \\
\hline Total bilirubin & & $0.3 \mathrm{mg} / \mathrm{dL}$ \\
\hline Lipid panel (mg/dL) & Levels in index patient & Reference range \\
\hline Total cholesterol & 155 & $<=200$ \\
\hline Triglycerides & 227 & $<=150$ \\
\hline LDL & 78 & $=>40$ \\
\hline $\mathrm{HDL}$ & 32 & $0-100$ \\
\hline
\end{tabular}

\section{TABLE 1: Labs at the time of admission.}

AST, aspartate aminotransferase; ALT, alanine transaminase; LDL, low-density lipoprotein; HDL, high-density lipoprotein

CT head did not reveal intracranial hemorrhage or mass. Arterial blood gas (ABG) was obtained and showed severe alkalosis with a pH of 7.72, with pCO2 $<20 \mathrm{mmHg}$. Serum bicarbonate was $28 \mathrm{mmol} / \mathrm{L}$ (Table 2).

\begin{tabular}{|c|c|c|}
\hline ABG & Preintubation & Postintubation \\
\hline $\mathrm{pH}$ & 7.72 & 7.47 \\
\hline PCO2 (mmHg) & $<20$ & 29 \\
\hline PO2 (mmHg) & 117 & 131 \\
\hline HCO3 (mmol/L) & Incalculable & 21 \\
\hline Base excess (mmol/L) & Incalculable & -2 \\
\hline aturation (\%) & 99 & 99 \\
\hline
\end{tabular}

TABLE 2: ABGs prior to intubation and postintubation.

$A B G$, arterial blood gas 


\section{Cureus}

Work-up for acute encephalopathy was done (Table 3).

\begin{tabular}{|c|c|c|}
\hline Lab & Level in index patient & Reference range \\
\hline UA & Negative for nitrite, leucocyte esterase, no wbc or bacteria & Negative \\
\hline Urine drug screen & Positive for benzodiazepines, opiates, oxycodone & Negative \\
\hline Ammonia (Mcmol/L) & 102 & $6-47$ \\
\hline Sed rate $(\mathrm{mmol} / \mathrm{L})$ & 8 & $0-15$ \\
\hline ANA titer & $<1: 80$ & $<1: 80$ \\
\hline VDRL syphilis & Nonreactive & Nonreactive \\
\hline Vitamin B12 (pg/mL) & 334 & $0-1000$ \\
\hline $\mathrm{TSH}(\mathrm{mcU} / \mathrm{mL})$ & 0.331 & $0.4-4.2$ \\
\hline T4 (ng/dL) & 0.8 & $0.6-1.5$ \\
\hline Folate $(\mathrm{ng} / \mathrm{mL})$ & 20.7 & $>=5.9$ \\
\hline Methylmalonic acid (Mcmol/L) & 0.13 & $0-0.4$ \\
\hline
\end{tabular}

TABLE 3: Work-up for acute encephalopathy and other pertinent labs.

UA, urinalysis; ANA, antinuclear antibody; VDRL, venereal disease research laboratory; TSH, thyroid stimulating hormone

MRI brain diffusion weighted images showed acute ischemic infarcts in bilateral cerebellar hemispheres, superior vermis, and PCA distribution (Figures 1-2).

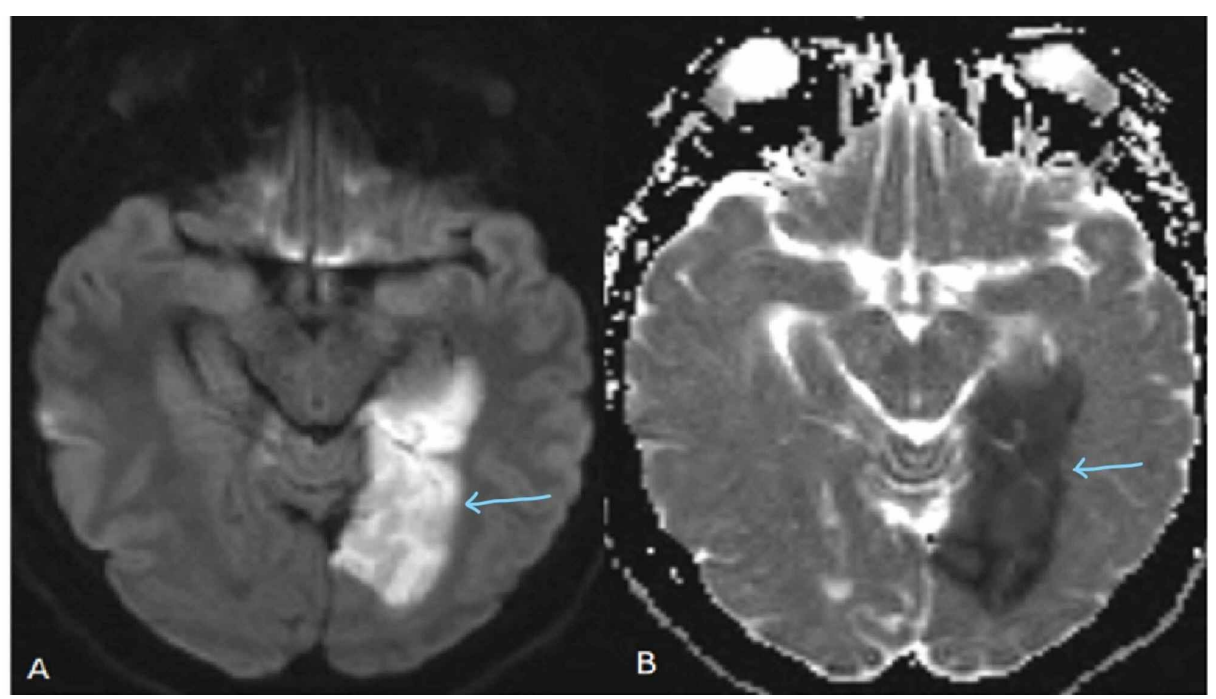

FIGURE 1: Diffusion weighted imaging (A) and corresponding ADC map (B) showing decreased diffusion in the left occipital lobe consistent with acute infarct (arrows pointing).

ADC, apparent diffusion coefficient 


\section{Cureus}

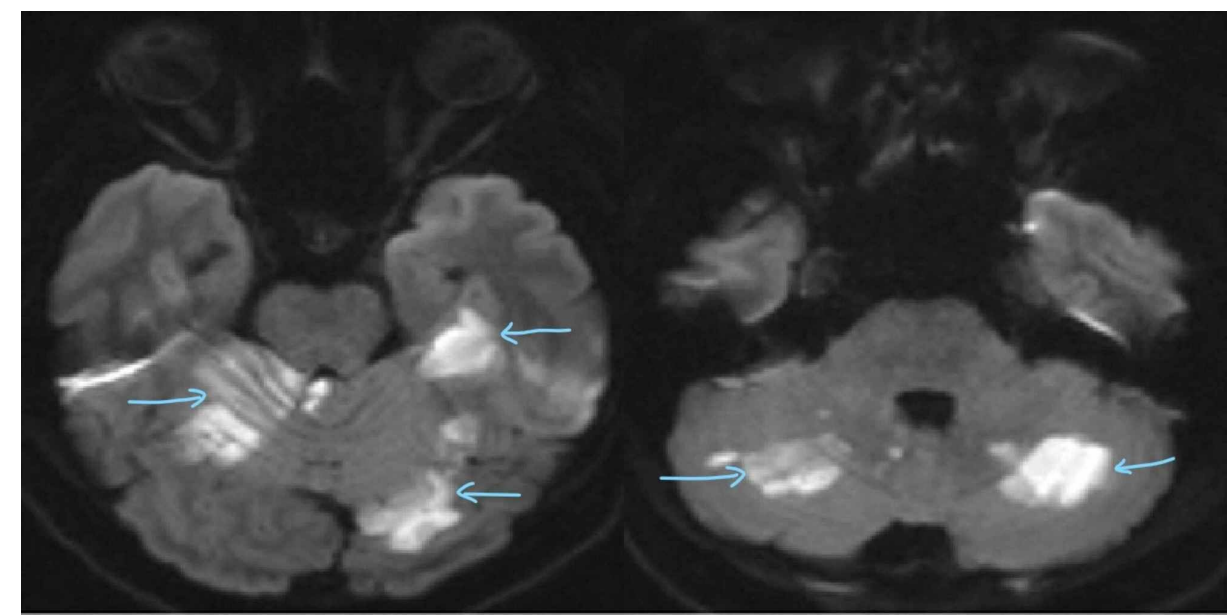

FIGURE 2: Diffusion weighted images show acute infarcts involving the left superior cerebellum (left side arrows) and bilateral inferior cerebellar hemispheres (right side arrows).

The CT angiogram of head and neck revealed a focal loss of gray-white differentiation involving the left temporal, occipital lobe in a left PCA distribution as well as a region in the left cerebellar hemisphere with no flow-limiting stenosis of the major arteries of the neck (Figure 3).



FIGURE 3: Maximum intensity projection of CT arteriography showing occlusion of a branch of the left posterior cerebral artery (arrow).

The patient was out of the therapeutic window for thrombolysis. The patient was started on aspirin, plavix, 
and high-intensity statin. Due to his altered mental status and severe respiratory alkalosis, he was transferred to ICU and intubated. Two hours postintubation, ABG showed improvement in the alkalosis (Table 2). The patient was extubated in two days. A transesophageal echocardiogram showed a patent foramen ovale (PFO). There was no evidence of venous thrombosis in lower extremities. No arrhythmias were seen on telemetry. He had some memory deficits but no motor or balance deficits by the time of discharge. The patient was discharged to a rehab facility in one week. He follows with the cardiology clinic at a tertiary center for the PFO closure.

\section{Discussion}

The presentation of AIS depends on the blood vessel involved and the area of ischemia. Our patient presented with an ischemic stroke with infarction in the bilateral cerebellar and left PCA territory. This is a case of cryptogenic AIS as the etiology of cerebral ischemia is not known despite work-up [4]. He was found to have a PFO, without evidence of venous thrombosis. Currently, he is following with cardiology regarding PFO closure, which has been shown to have better outcomes than medical therapy in his age group [7-8].

The patient became encephalopathic due to the stroke leading to severe respiratory alkalosis. ABG revealed pH 7.72, PCO2 < $20 \mathrm{mmHg}$, and serum bicarbonate $28 \mathrm{mmol} / \mathrm{L}$. The ABG is suggestive of acute primary and uncompensated respiratory alkalosis along with metabolic alkalosis. Respiratory alkalosis is the dominant acid base issue. The mild metabolic alkalosis was a result of vomiting. Vomiting causes metabolic alkalosis due to loss of hydrogen ion and hypovolemia [9]. But the dominant acid base abnormality in this case is primary respiratory alkalosis. Respiratory alkalosis is due to episodes of hyperventilation or sustained tachypnea/hyperpnea [10]. The index patient was hyperventilating at a rate of 18-26 at the time of presentation. This explains the respiratory alkalosis in the index case.

Based on the location of infarct on imaging, it is clear that the index patient suffered stroke in the left PCA and bilateral superior cerebellar artery territories. Ischemic stroke has been studied to cause respiratory alkalosis [10]. Various studies sought to explain the respiratory alkalosis in acute stroke. Primary central neurogenic hyperventilation has been suggested as a rare entity of hyperventilation without underlying pulmonary etiology and occurs despite alkalosis and low PCO2 [11]. The respiratory center has long known to reside predominantly in the brainstem, in pons and medulla [12]. Studies have been done recently to explore more about the respiratory centers in the nervous system [12]. Rowat et al. (2007) reported that respiratory control in the brain might be more spread out rather than reside in a single center. Furthermore, they also point out the possible role of humoral agents released from infarcted areas that influence respiratory pattern [13]. Through these studies, it is becoming more evident that respiratory and breathing patterns may not be related to one particular location or severity of stroke [14].

Respiratory pattern disturbances, including central periodic breathing (CPB)/Cheyne-Stokes Breathing, have been associated with acute stroke [15-16]. Cheyne-Stokes Breathing is a pattern of hypoventilation alternating with hyperventilation and has not been associated with any particular location of stroke [13-14]. Lee et al. noticed respiratory alkalosis in almost all stroke patients with Cheyne-Stokes Breathing pattern [16]. Kim et al. noticed that Cheyne-Stokes Respiration occurs irrespective of the location or severity of stroke [14]. Although, as per Rowat et al., Cheyne-Stokes Breathing is associated more with anterior circulation strokes [15].

Posterior circulation is divided into proximal (medulla and posterior inferior cerebellum), middle (pons and anterior inferior cerebellum), and distal (rostral brain stem, superior cerebellum, occipital and temporal lobes) [17]. The index patient had a stroke in the distal posterior circulation territory. Distal posterior circulation includes supply to rostral brainstem. Some brain stem involvement could have been possible and has been suggested by Anderson and Henrich in partial territory strokes [18].

There are a couple of salient but exciting features about our case. To our knowledge, such a high pH (> 7.7) associated with ischemic stroke has not been reported in the medical literature so far. There a few cases reported with a $\mathrm{pH}>7.7$ due to causes other than stroke. Also, we did not come across a respiratory alkalosis (plus mild metabolic alkalosis) with such a high $\mathrm{pH}$ as the cases with severe alkalosis previously reported are almost all due to metabolic alkalosis. Severe alkalosis causes metabolic disorders and several severe and detrimental complications to the patient's health [19]. A study by Sur and Shah found that patients with respiratory and metabolic alkalosis had a poor outcome, about $44.2 \%$ mortality [20]. In our case, early diagnosis and management of respiratory alkalosis resulted in the survival and recovery of the patient. He was not found to have any significant deficits during the post-recovery and follow-up period.

\section{Conclusions}

This case report highlights the potential, rare, but severe complication of respiratory alkalosis with AIS. The patient was successfully managed and has survived. Clinicians should keep this in mind in patients with AIS presenting with severe acute encephalopathy. Early diagnosis and management are crucial in survival and recovery. 


\section{Additional Information \\ Disclosures}

Human subjects: Consent was obtained by all participants in this study. Conflicts of interest: In compliance with the ICMJE uniform disclosure form, all authors declare the following: Payment/services info: All authors have declared that no financial support was received from any organization for the submitted work. Financial relationships: All authors have declared that they have no financial relationships at present or within the previous three years with any organizations that might have an interest in the submitted work. Other relationships: All authors have declared that there are no other relationships or activities that could appear to have influenced the submitted work.

\section{References}

1. Go AS, Mozaffarian D, Roger VL, et al.: Heart disease and stroke statistics--2013 update: a report from the American Heart Association. Circulation. 2013, 127:e6-e245. 10.1161/CIR.0b013e31828124ad

2. Sacco RL, Scott K, Joseph PB, et al.: An updated definition of stroke for the 21st century: a statement for healthcare professionals from the American Heart Association/American Stroke Association. Stroke. 2013, 44:2064-2089. 10.1161/STR.0b013e318296aeca

3. Adams HP, Bendixen BH Kappelle LJ, et al.: Classification of subtype of acute ischemic stroke. Definitions for use in a multicenter clinical trial. TOAST. Trial of Org 10172 in Acute Stroke Treatment. Stroke. 1993, 24:35-41. 10.1161/01.str.24.1.35

4. Finsterer J: Management of cryptogenic stroke. Acta Neurol Belg. 2010, 110:135-147.

5. Huang R: [Acid-base imbalance in acute cerebrovascular diseases] . Zhonghua Shen Jing Jing Shen Ke Za Zhi. 1991, 24:355-357, 384.

6. Hopkins E, Sharma S: Physiology Acid Base Balance. StatPearls Publishing, Treasure Island, FL; 2020.

7. Saver JL, John C, David T, et al.: Long-term outcomes of patent foramen ovale closure or medical therapy after stroke. N Engl J Med. 2017, 377:1022-1032. 10.1056/NEJMoa1610057

8. Turc G, David C, Patrice G, et al.: Closure, anticoagulation, or antiplatelet therapy for cryptogenic stroke with patent foramen ovale: systematic review of randomized trials, sequential meta-analysis, and new insights from the CLOSE study. J Am Heart Assoc. 2018, 7: 10.1161/JAHA.117.008356

9. Gilliion V, Jadoul M, Devuyst O, et al.: The patient with metabolic alkalosis. Int J Clin Lab Med. 2019, 74:3440.

10. Asplund. K, Fugl-Meyer AR, Engde M, et al.: Respiratory alkalosis early after stroke: its relation to locomotor function. Scand J Rehabil Med Suppl. 1983, 9:103-107.

11. Rowat AM, Dennis MS, Wardlaw JM: Central periodic breathing observed on hospital admission is associated with an adverse prognosis in conscious acute stroke patients. Cerebrovasc Dis. 2006, 21:340-347. $10.1159 / 000091540$

12. Lee MC, Klassen AC, Resch JA: Respiratory pattern disturbances in ischemic cerebral vascular disease. Stroke. 1974, 5:612-616. 10.1161/01.str.5.5.612

13. Rowat AM, Wardlaw JM, Dennis MS: Abnormal breathing patterns in stroke: relationship with location of acute stroke lesion and prior cerebrovascular disease. J Neurol Neurosurg Psychiatry. 2007, 78:277-279. 10.1136/jnnp.2006.102228

14. Kim Y, Kim S, Ryu DR, et al.: Factors associated with Cheyne-Stokes Respiration in acute ischemic stroke . J Clin Neurol. 2018, 14:542-548. 10.3988/jen.2018.14.4.542

15. Rowat AM, Dennis MS, Wardlaw JM: Central periodic breathing observed on hospital admission is associated with an adverse prognosis in conscious acute stroke patients.. Cerebrovasc Dis. 2006, 21:340-347. $10.1159 / 000091540$

16. Lee MC, Klassen AC, Resch JA: Respiratory pattern disturbances in ischemic cerebral vascular disease. AHA J. 1974, 5:612-616.

17. Caplan L, Chung CS, Wityk R, et al.: New England medical center posterior circulation stroke registry: I. Methods, data base, distribution of brain lesions, stroke mechanisms, and outcomes. J Clin Neurol. 2005, 1:14-30. 10.3988/jcn.2005.1.1.14

18. Anderson LE, Henrich WL: Alkalemia-associated morbidity and mortality in medical and surgical patients . South Med J. 1987, 80:729-733. 10.1097/00007611-198706000-00016

19. Amarenco P: Cerebellar infarctions and their mechanisms. Rev Neurol (Paris). 1993, 149:728-748.

20. Sur M, Shah AD: Alkalosis. StatPearls Publishing, Treasure Island, FL; 2020. 